\title{
Induction and evaluation of inflammatory markers by zinc oxide nanoparticles: A comparative study using in vitro and in vivo approaches.
}

\author{
Venkatachalam Deepa Parvathi ${ }^{1 *}$, Janani Boominathan ${ }^{1}$, Swetha SridharKumar ${ }^{1}$, Ravindran Sumitha ${ }^{2}$ \\ ${ }^{1}$ Department of Human Genetics, College of Biomedical Sciences, Technology and Research, Sri Ramachandra Institute \\ of Higher Education and Research, Porur, Chennai, Tamil Nadu, India \\ ${ }^{2}$ Department of Biomedical Sciences, Sri Ramachandra Institute of Higher Education and Research, Porur, Chennai, \\ India
}

\begin{abstract}
Zinc oxide nanoparticle is one of the most researched types of metal oxide nanoparticles in recent literature. Due to its unique properties such as biodegradability and reduced toxicity compared to other types of metallic nanoparticles, the application of zinc oxide nanoparticles has been increased in recent years. Most notable application is in the field of nanomedicine. Due to its ability to selectively kill cancer cells, zinc oxide nanoparticle has been used as a drug carrier for many chemotherapeutic drugs. The In vitro and in vivo models help us to understand the mechanism and the amount of toxicity induced by these nanoparticles in the immune system of humans. This can help in the formulation of personalized chemotherapeutic drugs. In this study, we analysed three differently synthesized zinc oxide nanoparticles (chemical precipitation, green chemistry approach, commercially available) on in vitro (human peripheral blood) and in vivo (Zebrafish) models. Pre-defined concentrations nanoparticles (5 $\mu \mathrm{g} / \mathrm{ml}, 10 \mu \mathrm{g} / \mathrm{ml}, 15 \mu \mathrm{g} / \mathrm{ml}, 20 \mu \mathrm{g} / \mathrm{ml}, 25 \mu \mathrm{g} / \mathrm{ml}$ and $30 \mu \mathrm{g} / \mathrm{ml}$ ) were used to analyse the amount of toxicity induced by zinc oxide nanoparticles. There was a dose dependent toxicity increase with in vitro and in vivo models after addition of zinc oxide nanoparticles. Commercially available zinc oxide nanoparticles showed highest toxicity and nanoparticles synthesized by green chemistry method showed lowest toxicity on comparison.
\end{abstract}

Keywords: Zinc oxide, Nanotoxicity, Nanomaterials, Haemolytic assay, ELISA.

Accepted on February 15, 2019

\section{Introduction}

\section{Nanomaterials}

Nanoscale materials are defined to have size between 1-100 $\mathrm{nm}$. There has been a tremendous increase in the application of nanoscale materials in various fields owing to their dynamic chemical and physical properties which make them more advantageous compared to their bulk counterparts. The unique properties help in the development of different devices in nanoplatforms that have variety of applications [1]. Of the many applications of nanoparticles in fields such as electronics, research, cosmetic industry the most important application is seen in nanomedicine that includes diagnosis, treatment and therapeutics for diseases like cancer. Nanoscale materials are widely used in drug delivery in nanomedicine.

The nanoscale materials can be classified into different groups such as inorganic, polymers and metal nanoparticles based on their physical and chemical properties [2].

Metal oxide nanoparticles have gained importance in therapeutics due to many advantages compared to other nanoscale particles. One of the most novel properties of metal oxide and metallic nanoparticle is the ability to generate reactive oxygen species in either the presence or absence of irradiation. This property is mainly exploited in nanomedicine in the form of drug delivery of chemotherapeutic drugs [3-5].

Zinc oxide nanoparticles have been extensively studied. Nano zinc has shown the ability to selectively kill tumor cells $[3,6]$. Other applications of zinc oxide nanoparticles include bioimaging, gene delivery, biosensing, drug delivery. The important property that makes zinc oxide nanoparticle more advantageous is its low toxicity and bio degradability [1,2].

Even though there is an increasing application of metal oxide nanoparticles in various fields, it's compatibility with the living tissue is considered as one of the major limitations in biomedical applications. Nanoscale substances of zinc oxide are classified as GRAS (generally recognised safe) by the FDA but any substance when reduced to nanoscale has the ability to exert new actions of toxicity. So, there is an importance of detailed evaluation of the toxicity of zinc oxide nanoparticles in both in vitro and in vivo systems. This can be helpful in reducing the toxicity induced $[4,7]$. 
In this study, we have measured the immunotoxicity, genotoxicity and cytotoxicity of differently synthesized nanoparticles in in vitro and in vivo systems. Nanotoxicity studies can be useful in defining the concentration and toxicity of nanoparticle that can be considered safe to be used in nanomedicine.

Exposure of the immune system to nanoparticles can result in either immunosuppression or immunostimulation. The primary recognition of nanoscale materials by the immune system can be a decisive component for deducing the effect of these nanoscale materials in the circulation after systemic distribution $[5,8]$.

In the present study, cytotoxicity and immunotoxicity of differently synthesized nanoparticles were done using various assays after exposure of peripheral blood to varied defined concentrations of nanoparticles.

(1) Haemolytic assay: Since, chemotherapeutic drugs interact with the cells of the circulatory system and RBC's are one of the most important and majorly occurring cells in the circulatory system, there is a possibility of haemolysis of RBC's on interaction with nanoparticles. Haemolysis is the process of rupture of cell membrane resulting in the decrease in oxygen binding capacity and increase in the pro oxidative effect $[6,7]$.

(2) MTT calorimetric assay: Cell based assays can be used to measure the cytotoxicity of a drug or the test compound. Here, the lymphocyte viability was measured after exposure of the cells to varied defined concentrations of nanoparticles. The percentage of viability can be used to deduce the amount of toxicity induced [7].

(3) Staining procedures: Cell viability and morphology can studied using viability assays such as trypan blue and acridine orange. The amount of live and dead cells present in the sample can be deduced by trypan blue assay that can be used to determine the cytotoxicity of nanoparticles. Acridine orange can be used to study the cellular morphology post exposure to nanoparticles [8].

(4) ELISA: One of the main properties of metal oxide nanoparticles is the induction of pro-inflammatory cytokines like TNF- $\alpha$ which plays an important role in inducing an immune response. The measure of release of pro inflammatory cytokines namely TNF- $\alpha$ can be used as an important measure of nanoimmunotoxicity [5]. ELISA was done in both in vitro and in vivo model to determine the immunotoxicity after exposure to varied defined concentrations of zinc oxide nanoparticles.

For in vivo studies, Zebrafish was used as model organism. Recent literatures suggest that there are many advantages of using Zebrafish for toxicity studies. Certain advantages include easy maintenance and breeding, small size and transparency of embryo making it a better model for toxicity studies [8-10].

In this study, nanotoxicity was measured by using different assays measuring genotoxicity, immunotoxicity and organ toxicity of Zebrafish after exposure to varied defined concentrations of zinc oxide nanoparticles.

(5) DNA fragmentation assay: Genotoxicity is the damage caused to the genetic content of the cell due to the exposure to harmful substances. The quality of DNA after exposure to zinc oxide nanoparticles can serve as an end point for toxicity measurements [8].

(6) Fin regeneration assay: Quick organ regeneration capacity of Zebrafish is one of the reasons for being the most used model organism. Although, fin is a complex appendage it has the ability to regenerate after amputation. Exposure to harmful substances can affect the regeneration capacity of Zebrafish which can be a useful measure of nanotoxicity $[9,10]$.

(7) Cardiotoxicity assay: One of the major concerns of drug trials is the organ toxicity. The development of heart in Zebrafish is rapid and has shown similarities with higher organisms. Number of heartbeats can be affected due to the exposure to zinc oxide nanoparticles and can be a measure to study the organ toxicity [9].

\section{Materials and Methods}

Three types of differently synthesized nanoparticles were used in this study.

\section{Synthesis of nanoparticles}

Zinc oxide nanoparticles synthesized by chemical precipitation method using zinc nitrate hexahydrate and sodium hydroxide as precursors [10]. Green chemistry approach was used to prepare zinc oxide nanoparticles where neem leaves (Azadirachta indica) was used as pre cursor material [11-14]. Commercially available zinc oxide nanoparticles were purchased from Sigma Aldrich (Lot \# MKBS3961V).

\section{Characterization}

Preliminary characterization was done using UV-Vis spectroscopy. The absorbance of zinc oxide nanoparticles was observed between 200-800 nm [11,13,14]. 3-6 ml of peripheral blood was used for in vitro study exposed to defined concentrations [12] of zinc oxide nanoparticles $(5 \mu \mathrm{g} / \mathrm{ml}, 10$ $\mu \mathrm{g} / \mathrm{ml}, 15 \mu \mathrm{g} / \mathrm{ml}, 20 \mu \mathrm{g} / \mathrm{ml}, 25 \mu \mathrm{g} / \mathrm{ml}$ and $30 \mu \mathrm{g} / \mathrm{ml})$. Juvenile and adult Zebrafish was used for in vivo study exposed to defined concentrations of zinc oxide nanoparticles ( $5 \mathrm{ppm}, 10$ ppm, 15 ppm, 20 ppm, 25 ppm, 30 ppm). Positive control (sample+Tween 20) and negative control (sample+DMSO) was used in the respective assays.

\section{In vitro assays}

Haemolytic assay: $200 \mu \mathrm{l}$ of $1 \%$ RBC suspension was exposed to defined concentrations of zinc oxide nanoparticles and a positive control and negative control. OD was calculated at $545 \mathrm{~nm}$ after 2 hours of exposure. Results were tabulated and graphically analysed based on the OD values. 
MTT calorimetric assay: $200 \mu \mathrm{l}$ of $1 \%$ WBC suspension was exposed to defined concentrations of zinc oxide nanoparticles and a positive control and negative control. OD was calculated at $540 \mathrm{~nm}$ after 2 hours of exposure. The OD values were tabulated and graphically analysed.

Staining assay: $200 \mu \mathrm{l}$ of $1 \%$ WBC suspension was exposed to defined concentrations of zinc oxide nanoparticles and a positive control and negative control. Trypan blue and acridine orange assays were performed to analyse the viability and morphology of exposed cells.

ELISA: $200 \mu \mathrm{l}$ of peripheral blood was exposed to defined concentrations of zinc oxide nanoparticles and a positive control and negative control. Serum was obtained after 2 hours of exposure. The ELISA protocol was followed according to Human Tumor Necrosis Factor Alpha ELISA kit (Bioassay Technology Laboratory Cat. No E0082Hu).

\section{In vivo assays}

DNA fragmentation assay: DNA was extracted from Zebrafish exposed to defined concentrations of zinc oxide nanoparticles and a control (Un-exposed fish) after 48 hours. To analyse the fragmentation DNA samples were run on a $3 \%$ agarose gel and results were documented $[15,16]$.

Fin regeneration assay: approximately $1 \mathrm{~mm}$ of caudal fin of zebra fish was amputated after proper anesthetization using a stereozoom microscope and the length of regenerated fin was measured after 5 days of exposure to defined concentrations of zinc oxide nanoparticles and a positive control and negative control.

Cardiotoxicity assay: Juvenile Zebrafish was exposed to defined concentrations of zinc oxide nanoparticles and a positive control and negative control. Number of heartbeats was calculated after 48 hours of exposure.

ELISA: Supernatant from homogenized sample of adult Zebrafish exposed to defined concentrations of zinc oxide nanoparticles and a positive control and negative control. The ELISA protocol was followed according to Human Tumor Necrosis Factor Alpha ELISA kit (Bioassay Technology Laboratory Cat. No E0082Hu).

\section{Results}

\section{Characterization of nanoparticles}

Preliminary characterization was done using UV-Vis spectroscopy. Double distilled water was used to dissolve the nanoparticles. Absorbance was seen at $373 \mathrm{~nm}$ for zinc oxide nanoparticle synthesized by chemical precipitation, $297 \mathrm{~nm}$ for zinc oxide nanoparticle synthesized by green chemistry approach and (sigma results). The absorbance between 200 $\mathrm{nm}-800 \mathrm{~nm}$ indicates the presence of nanoscale particles of zinc oxide in the sample [15-17].

Chemical composition of the nanoparticle was studied using SEM EDAX and results show no impurities in the nanoparticles synthesized by chemical precipitation. Nanoparticles synthesized by green chemistry approach showed the presence of potassium along with zinc and oxygen. This cannot be inferred as an impurity because potassium is present in neem leaves naturally. The fraction of $\mathrm{ZnO}$ nanoparticles obtained through EDAX was subjected to SEM analysis (both chemical and green synthesis). The size of the nanoparticle was studied using SEM and was found to be in the nanoscale. SEM analysis showed that the size of the nanoparticles were in the range of $12-19 \mathrm{~nm}$ for chemically synthesised and 22-30 nm for green chemistry method [18-21].

\section{In vitro assays}

Haemolytic assay: The absorbance post exposure is a measure of cytotoxicity induced by synthesized $\mathrm{ZnO}$ nanoparticles. The amount of lysis was calculated with exposure to defined concentrations. The results were analysed on comparison with positive and negative control. There was a dose dependent increase in lysis observed. Highest lysis was observed in 30 $\mu \mathrm{g} / \mathrm{ml}$. Highest toxicity was induced by commercially available nanoparticles and lowest toxicity was induced by nanoparticles synthesized by green chemistry approach [6,22-26].

MTT calorimetric assay: MTT assay was performed to determine the cell viability in the sample post exposure. The results were calculated with respect to negative control. A dose dependent reduction in cell viability was observed, lowest viability was observed in sample exposed to $30 \mu \mathrm{g} / \mathrm{ml}$. Highest toxicity was induced by commercially available nanoparticles and lowest toxicity was induced by nanoparticles synthesized by green chemistry approach $[25,26]$.

Staining assays: Trypan blue staining was done to determine cell viability in the sample post exposure to defined concentrations (5 $\mu \mathrm{g} / \mathrm{ml}, 10 \mu \mathrm{g} / \mathrm{ml}, 15 \mu \mathrm{g} / \mathrm{ml}, 20 \mu \mathrm{g} / \mathrm{ml})$. The results were analysed on comparison with positive and negative control (suspension+DMSO). Correlating to results obtained in MTT there was a dose dependent increase in amount of non-viable cells and decrease in the amount of viable cells in the sample. Lowest viability was seen in sample exposed to $30 \mu \mathrm{g} / \mathrm{ml}$. Highest toxicity was induced by commercially available nanoparticles and lowest toxicity was induced by nanoparticles synthesized by green chemistry approach.

Cellular morphology was analysed in the samples exposed to varied defined concentrations. The results were analysed on comparison with positive and negative control. There was a dose dependent increase in staining intensity and damage to the exposed cells that was observed. Also, nuclear blebbing was clearly seen in cells exposed to commercially available zinc oxide nanoparticles and cells exposed to positive control.

ELISA TNF- $\alpha$ : Pro inflammatory cytokine TNF- $\alpha$ concentration was determined as a measure of immunotoxicity of sample exposed to defined concentrations. There was a dose dependent increase in the concentration of TNF- $\alpha$ in PBMC in the exposed samples. Highest fold was seen in $30 \mu \mathrm{g} / \mathrm{ml}$. Also, the TNF- $\alpha$ concentration was seen lowest in nanoparticles 
synthesized by chemical precipitation compared to other nanoparticles.

\section{In vivo assays}

DNA fragmentation assay: this assay was done as a measure of genotoxicity induced in Zebrafish exposed to varied defined concentrations. Results showed considerable shearing or fragmentation of DNA compared to control sample in all the three types of nanoparticles.

Fin regeneration assay: this procedure was done as a measure of organ toxicity post exposure to varied defined concentrations of zinc oxide nanoparticles. The results were tabulated with respect to positive and negative control. There was dose dependent decrease in length of fin regenerated. Highest toxicity was induced by commercially available nanoparticles and lowest toxicity was induced by nanoparticles synthesized by green chemistry approach.

Cardiotoxicity assay: Cardiotoxicity was done as an assessment of organ toxicity. Juvenile fishes exposed to varied defined concentrations of nanoparticles were assessed for number of heartbeats post exposure. The results showed that there was a significant dose dependent decrease in heartbeats observed per minute. Highest toxicity was induced by commercially available nanoparticles and lowest toxicity was induced by nanoparticles synthesized by green chemistry approach [20,21].

ELISA TNF- $\boldsymbol{\alpha}$ : Pro-inflammatory responses to varied defined concentrations to nanoparticles were measured by ELSA. There was dose dependent increase in the concentration of TNF- $\alpha$ induced by $\mathrm{ZnO}$ nanoparticles. Highest fold was seen in 20 ppm. The TNF- $\alpha$ concentration was seen lowest in nanoparticles synthesized by chemical precipitation compared to other nanoparticles [22-24].

\section{Discussion}

Nanotoxicity is one of the important concerns of nanoscale materials. Simpler assays measuring genotoxicity, cytotoxicity, organ toxicity and immunotoxicity of nanoparticles can help in better understanding on behaviour of nanomaterials with the immune system and also in formulating personalised nanodrug [22-27]. As the literature suggests, toxicity also depends on the morphology and chemical composition of the nanomaterials $[17,18]$. The methods used for synthesis in the study proved to be simpler, cost effective and giving more yield with no impurities. Concentrations defined in this study were defined according to the literature stating $10 \mu \mathrm{g} / \mathrm{ml}$ as baseline toxicity. Cytotoxicity assays such as haemolytic assay, MTT assay showed a dose dependent increase in toxicity and highest toxicity was absorbed in $30 \mu \mathrm{g} / \mathrm{ml}$. Viability assays such as trypan blue showed dose dependent increase in the number of non-viable cells and decrease in the number of viable cells as the concentration increased. Acridine orange assay showed considerable damage in the exposed cells. Interestingly, nuclear blebbing was clearly seen in cells exposed to commercially available zinc oxide nanoparticles and cells exposed to positive control. Pro-inflammatory cytokine TNF- $\alpha$ expression measured using ELISA showed a dose dependent increase concentration of TNF- $\alpha$ and highest fold was seen in $30 \mu \mathrm{g} / \mathrm{ml}$ [25]. Genotoxicity was analysed by DNA fragmentation assay done on in vivo model Zebrafish showed considerable fragmentation/shearing of DNA after exposure compared to un exposed control. Organ toxicity was analysed using cardiotoxicity and fin regeneration assays that showed a dose dependent increase in damage caused due to the exposure to nanoparticles. The results were consistent with research studies conducted "hitherto'. Comparing the toxicity between differently synthesized nanoparticles showed that nanoparticles synthesized by green chemistry approach induced lowest toxicity and commercially available zinc oxide nanoparticles induced highest toxicity. Interestingly, only the TNF- $\alpha$ concentration was seen lowest in nanoparticles synthesized by chemical precipitation compared to other nanoparticles. Thus, the assays used in the present study could be a prelude to understanding the mechanism behind the induction of toxicity and can help in determining concentrations of usage of nanoscale materials in biomedical and therapeutic applications [27-29].

\section{Declaration of Conflict of Interest}

The author(s) declare(s) that there is no conflict of interest regarding the publication of this paper.

\section{Data Availability Statement}

The Data (Images, Figures and Tables) used to support the findings of this study are available from the corresponding author upon request. The same can also be made available as supplementary files if required.

\section{Funding Statement}

This research was conducted as a part of Masters Dissertation. This research did not receive specific funding, but was performed as part of the employment and period of study of the authors at the Department of Human Genetics, Sri Ramachandra Institute of Higher Education and Research, Chennai, India.

\section{References}

1. Zhang Y, Nayak TR, Hong H, Cai W. Biomedical applications of zinc oxide nanomaterials. Curr Mol Med 2013; 13: 1633-1645.

2. Khanna P, Ong C, Bay BH, Baeg GH. Nanotoxicity: an interplay of oxidative stress, inflammation and cell death. Nanomaterials (Basel) 2015; 5: 1163-1180.

3. Augustine R, Mathew AP, Sosnik A. Metal oxide nanoparticles as versatile therapeutic agents modulating cell signaling pathways: linking nanotechnology with molecular medicine. Appl Mater Today 2017; 7: 91-103.

4. Rasmussen JW, Martinez E, Louka P, Wingett DG. Zinc oxide nanoparticles for selective destruction of tumor 
in vivo approaches

cells and potential for drug delivery applications. Expert Opin Drug Deliv 2010; 7: 1063-1077.

5. Hussain S, Vanoirbeek JA, Hoet PH. Interactions of nanomaterials with the immune system. Wiley Interdiscip Rev Nanomed Nanobiotechnol 2012; 4: 169-183.

6. Preedia Babu E, Subastri A, Suyavaran A, Premkumar K, Sujatha V, Aristatile B. Size dependent uptake and hemolytic effect of zinc oxide nanoparticles on erythrocytes and biomedical potential of $\mathrm{ZnO}$-ferulic acid conjugates. Sci Rep 2017; 7: 4203.

7. Riss TL, Moravec RA, Niles AL, Duellman S, Benink HA, Worzella TJ. Cell viability assays. Assay Guidance Manual. Bethesda (MD): Eli Lilly and Company and the National Center for Advancing Translational Sciences 2013.

8. Hanley C, Layne J, Punnoose A, Reddy KM, Coombs I, Coombs A. Preferential killing of cancer cells and activated human $\mathrm{T}$ cells using $\mathrm{ZnO}$ nanoparticles. Nanotechnology 2008; 19: 295103.

9. Ma C, Parng C, Seng WL, Zhang C, Willett C, McGrath P. Zebrafish: an in vivo model for drug screening. Innov Pharmaceut Tech 2003; 38-45.

10. Suntako R. Effect of zinc oxide nanoparticles synthesized by a precipitation method on mechanical and morphological properties of the CR foam. Bull Mater Sci 2015; 38: 1033-1038.

11. Elumalai K, Velmurugan S. Green synthesis, characterization and antimicrobial activities of zinc oxide nanoparticles from the leaf extract of Azadirachta indica (L.). Appl Surf Sci 2015; 345: 329-336.

12. Andersson-Willman B, Gehrmann U, Cansu Z, BuerkiThurnherr T, Krug HF, Gabrielsson S, Scheynius A. Effects of subtoxic concentrations of $\mathrm{TiO} 2$ and $\mathrm{ZnO}$ nanoparticles on human lymphocytes, dendritic cells and exosome production. Toxicol Appl Pharmacol 2012; 264: 94-103.

13. Wang L, Muhammed M. Synthesis of zinc oxide nanoparticles with controlled morphology. J Mater Chem 1999; 9: 2871-2878.

14. Becheri A, Durr M, Nostro PL, Baglioni P. Synthesis and characterization of zinc oxide nanoparticles: application to textiles as UV-absorbers. J Nanopart Res 2008; 10: 679-689.

15. Talam S, Karumuri SR, Gunnam N. Synthesis, characterization, and spectroscopic properties of $\mathrm{ZnO}$ nanoparticles. ISRN Nanotechnol 2012; 2012.

16. Panigrahi S, Kundu S, Ghosh S, Nath S, Pal T. General method of synthesis for metal nanoparticles. J Nanopart Res 2004; 6: 411-414.

17. Chen C, Liu P, Lu C. Synthesis and characterization of nano-sized $\mathrm{ZnO}$ powders by direct precipitation method. Chem Eng J 2008; 144: 509-513.

18. Suwanboon S, Amornpitoksuk P, Haidoux A, Tedenac JC. Structural and optical properties of undoped and aluminium doped zinc oxide nanoparticles via precipitation method at low temperature. J Alloys Compounds 2008; 462: 335-339.

19. Kumar SS, Venkateswarlu P, Rao VR, Rao GN. Synthesis, characterization and optical properties of zinc oxide nanoparticles. Int Nano Lett 2013; 3.

20. Ma C, Parng C, Seng WL, Zhang C, Willett C, McGrath P. Zebrafish: an in vivo model for drug screening. Innov Pharmaceut Tech 2003; 38-45.

21. Gemberling M, Bailey TJ, Hyde DR, Poss KD. The zebrafish as a model for complex tissue regeneration. Trends Genet 2013; 29: 611-620.

22. Zolnik BS, Gonzalez-Fernandez A, Sadrieh N, Dobrovolskaia MA. Minireview: Nanoparticles and the Immune System. Endocrinology 2010; 151: 458-465.

23. Dobrovolskaia MA, McNeil SE. Immunological properties of engineered nanomaterials. Nat Nanotechnol 2007; 2: 469.

24. Hussain S, Vanoirbeek JAJ, Hoet PHM. Interactions of nanomaterials with the immune system. Wiley Interdiscip Rev Nanomed Nanobiotechnol 2011; 4: 169-183.

25. Huang H, Lai W, Cui M, Liang L, Lin Y, Fang Q, Liu Y, Xie L. An evaluation of blood compatibility of silver nanoparticles. Sci Rep 2016; 6: 25518.

26. Preedia Babu E, Subastri A, Suyavaran A, Premkumar K, Sujatha V, Aristatile B, Alshammari GM, Dharuman V, Thirunavukkarasu C. Size dependent uptake and hemolytic effect of zinc oxide nanoparticles on erythrocytes and biomedical potential of $\mathrm{ZnO}$-ferulic acid conjugates. Sci Rep 2017; 7: 4203.

27. Raghupathi KR, Koodali RT, Manna AC. Size-dependent bacterial growth inhibition and mechanism of antibacterial activity of zinc oxide nanoparticles. Langmuir 2011; 27: 4020-4028.

28. Niaz K, Khan F, Abdollahi M. Toxicity of biologically active peptides and future safety aspects: an update. Curr Drug Discov Technol 2018; 15.

29. Kourosh K, Benjamin F. Characterization techniques for nanomaterials. Nanotechnology-Enabled Sensors 2008; 211-281.

\section{*Correspondence to}

Deepa Parvathi V

Department of Human Genetics

College of Biomedical Sciences, Technology and Research

Sri Ramachandra Institute of Higher Education and Research

Tamil Nadu

India 